\title{
Salivary Metabolic Hormones as Biomarkers of Childhood Obesity
}

\section{Shiny Lizia Manohar}

Sri Ramachandra Institute of Higher Education and Research

Hemamalini Arambakkam Janardhanam ( $\nabla$ sruphd391@gmail.com )

Sri Ramachandra Institute of Higher Education and Research https://orcid.org/0000-0002-9993-5003

\section{Latha Ravichandran}

Sri Ramachandra Institute of Higher Education and Research

\section{Research Article}

Keywords: Saliva, Appetite, Metabolism, Hormones, Childhood Obesity

Posted Date: October 13th, 2021

DOI: https://doi.org/10.21203/rs.3.rs-446419/v2

License: (c) (1) This work is licensed under a Creative Commons Attribution 4.0 International License. Read Full License 


\section{Abstract}

\section{Introduction}

Obesity is a major threat to the health and development of children. While hormones in serum samples have been extensively investigated in childhood obesity research, our study attempts to explore salivary metabolic hormones as biomarkers of childhood obesity.

\section{Methods}

Obese $(n=83)$ and non-obese $(n=83)$ school children aged 6-11 years were recruited as cases and controls, respectively, for this case-control study. Salivary concentrations of the hormones, Ghrelin, Leptin, Adiponectin and Insulin were assessed and compared between obese and non-obese children to evaluate their role in childhood obesity.

\section{Results}

The mean ghrelin $(1.69 \pm 0.38 \mathrm{pg} / \mathrm{ml})$ and adiponectin $(95.53 \pm 64.92 \mathrm{ng} / \mathrm{ml})$ levels of the non-obese children were higher than the mean values of the obese, while the mean values of the hormones leptin $(265.95 \pm 83.16 \mathrm{pg} / \mathrm{ml})$ and insulin $(224.56 \pm 184.86 \mathrm{pg} / \mathrm{ml})$ were found to be higher among the obese. Higher levels of the hormones insulin (OR: 1.09; 95\% Cl: 1.05-1.12) and leptin (OR: 1.03; 95\% Cl: 1.021.04) posed higher risks for childhood obesity, followed by adiponectin, which was found to be lower among the cases (OR: 0.99; $95 \% \mathrm{Cl}$ : 0.99995-0.99998) which increased the risk of childhood obesity.

\section{Conclusion}

This study demonstrates the efficacy of saliva as a promising non-invasive tool in childhood obesity research and highlights the significance of appetite regulatory hormones as biomarkers of childhood obesity.

\section{Introduction}

Childhood obesity is a public health burden worldwide. It is alarming to see the increasing rates of childhood obesity in developing countries like India. Childhood subsists to be a sensitive period for neurological, endocrine, and metabolic development. Obesity evolving at a young age contributes to an increased risk of diabetes, hypertension, and cardiovascular disease in adulthood [1]. Obesity is a multifaceted problem with many contributing factors including genetics, hormone levels, overconsumption of food and sedentary lifestyle. Appetite regulation is a complex process involving communication between the hypothalamus within the brain, various gastrointestinal organs (including the stomach, the pancreas, and the intestines) and adipose tissue. Satiation (the signal that causes one to stop eating) may be initiated by neural input from the stomach to the brain, signaling gastric distension 
after food consumption. This is quickly followed by the release of various hormones sensing the digestion and absorption of nutrients and initiate satiety (the feeling of fullness that persists after eating). The gastrointestinal tract is the largest endocrine organ in the body and plays an important appetite-regulating role as a source of numerous regulatory peptide hormones. When the levels of the hormones inducing hunger increase, the demand for food intake increases, thereby contributing to excess energy storage and increased levels of fat, all of which sum up to the cause of obesity [2].

While most of the metabolic biomarker analysis in the field of obesity is performed using blood samples, studies on salivary biomarker analysis seem to be scarce. The salivary proteome consists of thousands of proteins, including hormonal mediators of energy balance. Studies state that human salivary glands can produce and release ghrelin and have also observed a significant correlation with serum or plasma ghrelin levels [3]. Saliva could be a boon in pediatric populations considering the parental apprehension towards withdrawal of blood samples from children. Salivary biomarkers have also been reported to be an effective and sustainable alternative due to the ease of tissue access, cost-efficiency and the ability to collect it in multiple settings, repetitively [4]. In comparison to collection of blood, saliva is associated with lower infection rates, decreased cost, increased patient acceptance, and higher participant compliance. Saliva also has the advantage of offering insight into the gastrointestinal tract, which could be useful when examining obesity [1].

Accordingly, in our study, we have adopted saliva as the medium of biomarker analysis among children. The biomarkers of interest were appetite regulatory hormones such as ghrelin, leptin, adiponectin and insulin. Although there are several studies in both children and adults on the causes of obesity, little is known about the physiological role of appetite regulatory hormones in humans, especially children. Hence, there is ample scope for research in this area [2]. In this pretext, the framework for our study was developed to evaluate the significance of salivary metabolic hormones as biomarkers of childhood obesity.

\section{Materials And Methods}

\section{Study Design}

A case-control study design was adopted in this study. Obese and non-obese school children of the age group (6-11 years) were the subjects of interest in this study. The levels of salivary metabolic hormones Ghrelin, Leptin, Adiponectin and Insulin in obese and non-obese subjects were assessed and compared to determine the significance of these hormones as biomarkers of childhood obesity.

\section{Ethical Approval}

All procedures performed in this study were in accordance with the ethical standards of the institutional and national research committee (Indian Council of Medical Research) and with the 1964 Helsinki Declaration and its later amendments or comparable ethical standards. The study was approved by the Institutional Ethics Committee of Sri Ramachandra Institute of Higher Education and Research (IEC- 
$\mathrm{NI} / 15 / \mathrm{FEB} / 45 / 07)$. Written informed consent was obtained from the parents of the children who were willing to allow their children participate in the study.

\section{Setting}

The study was conducted in an elementary school and a summer campsite in Chennai, India, from July 2017 to March 2018, as part of an extensive research in the area of salivary appetite regulatory hormones and an intervention program targeting these hormones in obese children. Permission was obtained from the concerned authorities of the elementary school and the summer campsite for conducting the study. Anthropometric assessment of all children aged between six and eleven years was carried out at the sample sites to categorize them into obese and non-obese groups. Children who gave written informed parental consent and assent to participate in the study alone were recruited for the study as per the sample size recruitment.

\section{Participants}

A total of 1432 children from an elementary school $(n=1378)$ and a summer campsite $(n=54)$ were assessed for childhood obesity, using standard protocols. Of the 1432, 166 children (83 obese and 83 non-obese) were recruited for this study based on inclusion and exclusion criteria and consent to participate in the study. The criteria for inclusion were - i) children aged between six and eleven years categorized as normal (control) and obese (case) using the WHO growth reference for school aged children (2007) and, ii)children who gave written informed parental consent and assent to participate in the study. The criteria for exclusion were - i) children who had an obvious underlying medical cause of obesity (evaluated using a questionnaire) and, ii) children who had undergone any medical or nutritional therapy for obesity in the past six months.

\section{Tools Used -}

\section{Anthropometry}

Anthropometric measurements such as height and body weight of the subjects were assessed using standard protocols. A calibrated stadiometer was mounted on a wall and the height (in centimeter (cm)) of each subject was measured in an upright standing position on an even surface adjacent to the wall. Body weight of each subject was measured using a body fat analyzer (TANITA UM-076) and recorded in kilogram $(\mathrm{kg})$. The readings were entered in the nutritional survey module of WHO AnthroPlus v.1.0.4 (2009) software. The height-for-age ' $Z$ ' (HAZ) scores, weight-for-age ' $Z$ ' (WAZ) scores, and body mass index-for-age ' $Z$ ' (BAZ) scores of the subjects were calculated using the WHO AnthroPlus software and the subjects were categorized into obese and non-obese categories based on their BAZ scores.

\section{Salivary Analysis}

\section{Collection of samples of Saliva}


On the day of sample collection, the subjects were asked to come to the sample site in a state of fasting. The subjects were made to rinse their mouth with water before sample collection. Saliva was collected using sublingual cotton roll technique (cotton roll placed under the tongue of the subject for a minute). Using a sterile forceps, the cotton roll was transferred to a $50 \mathrm{~mL}$ syringe and injected into a vacutainer tube, to collect approximately two milliliter of saliva from each subject. The samples collected in vacutainer tubes were arranged in a 96-vial storage rack placed in a freezer box. The principle investigator shifted the samples stored in the freezer box to the testing laboratory under safe conditions within 24 hours of sample collection. The samples were stored at $-20^{\circ} \mathrm{C}$ and analyzed within a period of six months.

\section{Multiplex Analysis of Salivary Markers}

Magnetic Luminex ${ }^{\circledR}$ Assays were used to assess the concentration of the selected hormones in each sample. The assays (166 saliva samples) for three biomarkers leptin, adiponectin and insulin were performed on $50 \mu$ of saliva sample using premixed 3-plex magnetic bead panels on a Bioplex 200 platform with no dilution. The assay procedure was carried out following the manufacturers' protocol. The results were read within 90 minutes post assay and evaluated using an analyzer.

\section{Ghrelin Assay}

As inclusion of ghrelin in the premixed kit used for the other markers was not possible due to the interference in magnetic bead regions, a separate test kit (RayBio® Ghrelin Enzyme Immunoassay (EIA) Kit: EIA-GHR, EIAM-GHR, EIAR-GHR) was used to assess ghrelin in $100 \mu \mathrm{l}$ of each saliva sample. The assay employed an in vitro quantitative technique for detecting ghrelin peptides based on competitive enzyme immunoassay principle. The assay procedure was carried out following the manufacturers' protocol. A standard curve of known concentration of Ghrelin peptide in the samples was assessed.

\section{Sample size}

166 (Obese, Cases $=83$; Non-obese, Controls $=83$ ) was the calculated sample size, assuming $\mu 1=48.0$ and $\mu 2=63.1$; Difference of means $=24.6$, based on the study conducted by Gil et al., 2009 [5], at a Power of $80 \%$. The sample size was calculated using Piface by Russell V. Lenth. Version $1.76-29$ June 2011.

\section{Statistical analysis}

All descriptive statistics are expressed using mean and standard deviation after analyzing the data for normality, confirming the normal distribution of data. Independent sample t-test was used to test the mean difference between the control and case groups. Box and whisker plots were drawn to display the variation in hormone levels of the subjects through the quartiles of data. Univariate binary logistic regression was used to calculate the risk factor analysis. Odds ratio was calculated to evaluate the odds

of salivary metabolic hormones as biomarkers among the obese and non-obese groups and to imply an 
association between the salivary hormones and childhood obesity. The statistical analyses were done using SPSS version 23.0 and any $p$ - value less than 0.05 was considered as statistically significant.

\section{Results}

Of the 1432 children assessed for enrollment in the study, 758 (256 obese and 502 non-obese) fulfilled the inclusion criteria, out of which 592 subjects were excluded from the study due to dissent to participate and randomized selection. Finally, a total of 166 subjects ( 83 obese and 83 non-obese) participated in the study and there were no drop outs, as the study involved a one-time diagnostic observation. Study report flowchart according to the STROBE (Strengthening the Reporting of Observational Studies in Epidemiology) is presented in Figure 1.

The mean values of baseline characteristics of the subjects are mentioned in table 1 . The mean age of the subjects was $8.65 \pm 1.06$ years. With respect to anthropometry, the mean HAZ of the subjects in control group and case group was found to be $135.07 \pm 7.14$ and $133.76 \pm 6.23$, respectively. The mean WAZ of the control group was found to be $0.07 \pm 0.94$ and that of the case group was $1.72 \pm 0.39$ ( $p$ value $<0.001)$. The mean BAZ of the subjects belonging to the control group was found to be $-0.13 \pm 0.93$ and that of the cases was found to be $2.11 \pm 0.28$ ( $p$ value $<0.001$ ).

Figure 2 pictorially represents the mean salivary concentrations of metabolic hormones of the subjects using box and whisker plots. Mean salivary ghrelin values of obese and non-obese children were, $0.77 \pm 0.28 \mathrm{pg} / \mathrm{ml}$ and $1.69 \pm 0.38 \mathrm{pg} / \mathrm{ml}$, respectively. Mean salivary adiponectin values of obese and nonobese groups were, $28.74 \pm 22.06 \mathrm{ng} / \mathrm{ml}$ and $95.63 \pm 65.92 \mathrm{ng} / \mathrm{ml}$, respectively. The mean concentrations of the hormones leptin among obese and non-obese groups were, $265.95 \pm 83.16 \mathrm{pg} / \mathrm{ml}$ and $136.99 \pm 72.48$ $\mathrm{pg} / \mathrm{ml}$, respectively. Mean salivary insulin concentrations of obese and non-obese children were, $224.56 \pm 184.86 \mathrm{pg} / \mathrm{ml}$ and $47.64 \pm 23.76 \mathrm{pg} / \mathrm{ml}$, respectively. The statistical evaluation of mean difference of salivary metabolic hormones between obese and non-obese groups revealed that there was a significant difference in the concentration of salivary biomarkers $(P<0.001)$.

The odds ratio is a measure of relative risk and is usually calculated by dividing the odds of exposure among the cases by the odds of exposure among the controls [6]. In this study, the exposure was considered to be salivary metabolic hormone concentrations, in order to investigate the odds of these hormones as risk factors for childhood obesity. The results of the univariate binary logistic regression analysis highlighted the significance of salivary metabolic hormones as biomarkers of childhood obesity. It reveals that, higher levels of the hormones insulin (OR: 1.09; 95\% Cl: 1.05-1.12) and leptin (OR: 1.03; $95 \% \mathrm{Cl}: 1.02-1.04)$ pose higher risks for childhood obesity, followed by adiponectin, which was found to be lower among the cases (OR:0.99; 95\% Cl:0.99995-0.99998) increasing the risk for childhood obesity (Table 2).

\section{Discussion}


The gut-brain axis harbors a pivotal role in the regulation of food intake and the maintenance of body weight. A complex array of signals from peripheral and central nervous systems, likely under epigenetic programming influences psychological and social factors to determine energy balance and body weight homeostasis [7]. The cluster of hormones that regulate appetite and food intake is wide-ranging. When the levels of the hormones inducing hunger increase, the demand for food intake increases, thereby contributing to excess energy storage and increased levels of fat, all of which sum up to the cause of obesity. Belfort-DeAguiar and Seo (2018) concluded that, obese individuals have elevated insulin and leptin levels and decreased ghrelin levels in comparison to normal weight individuals [8]. They have affirmed that insulin and leptin levels parallel body weight status, and insulin and leptin resistance play an indicative role in the pathogenesis of obesity. Thanakun et al., (2014) and Li et al., (2010) found significant correlation between salivary and serum ghrelin and adiponectin levels, suggesting salivary ghrelin could be a possible alternative to serum ghrelin as a biomarker in predicting the risk of childhood obesity $[9,10]$.

In our study we observed a significant difference in the concentration of salivary metabolic hormones among the obese and non-obese subjects. The results indicated that the mean concentrations of ghrelin and adiponectin of non-obese group were higher than the mean values of obese, while the mean values of the hormones leptin and insulin were found to be higher among obese when compared to the nonobese controls. Several studies on serum analysis of appetite hormones highlight that obese children show significant lower adiponectin and ghrelin concentrations and higher insulin and leptin levels [11-13]. The findings of the study conducted by Goodson et al., (2014) on metabolic disease risk in children using salivary biomarkers were consonant with the results of ours, presenting lower levels of ghrelin and adiponectin in the obese group when compared to the normal subjects and higher levels of insulin and leptin in the obese group [14].

The mechanism behind the downregulation of ghrelin in obese children could be attributed to their elevated leptin or insulin levels, as studies show that fasting ghrelin levels negatively correlate with fasting insulin and leptin levels. This state represents an adaptation towards positive energy balance and increased weight gain in these children [15]. Decreased concentration of adiponectin among the obese children in our study is similar to the findings of many studies carried out in the serum samples of obese children [16-18]. Adiponectin may be one of the signals linking inflammation and obesity. Soliman et al., (2012) suggest that in a majority of obese individuals, serum leptin concentrations are increased and leptin administration shows only very limited effects due to leptin resistance [19]. Leptin resistance is associated with insulin resistance and abdominal obesity. Increased appetite is associated with altered levels of appetite regulatory hormones and thus, these hormones are identified as potential neuroendocrine markers and mediators in childhood obesity, as insisted by Hagen et al., (2015) [20].

\section{Conclusion}

Despite the need for robust research in childhood obesity, the practical challenges and risks in including children being vulnerable groups, as subjects, limit the scope of clinical research in this field. While 
difficulty in obtaining permissions from authorities and parents for using invasive techniques like blood withdrawal are on one hand, on the other, convincing children to such invasive methods pose a great challenge. Such difficulties form one of the major reasons for dropouts from the study. Hence, the conquest for reliable, sustainable and effective non-invasive tools of clinical research is of dire need. Saliva seems to be a promising non-invasive tool to widen the scope of clinical research in childhood obesity. The present study has thrown light on the possibility of considering salivary samples in the evaluation of metabolic hormones, which may further enable us to intervene and prevent or treat the onset or incidence of childhood obesity.

\section{Declarations}

\section{Acknowledgement}

The authors extend their gratitude to the study participants and the school management for their cooperation. They wish to thank Dr. Sheila John, Associate Professor and Head (PG \& Research Department - Department of Home Science) \& Vice-Principal, Women's Christian College, Chennai, India, for offering her valuable expertise and support during the conduct of the study.

\section{Statement of Ethics}

All procedures performed in this study were in accordance with the ethical standards of the institutional and national research committee (Indian Council of Medical Research) and with the 1964 Helsinki Declaration and its later amendments or comparable ethical standards. The study was approved by the Institutional Ethics Committee of Sri Ramachandra Institute of Higher Education and Research (IEC$\mathrm{NI} / 15 / \mathrm{FEB} / 45 / 07)$. Written informed consent was obtained from the parents of the children who were willing to allow their children participate in the study.

\section{Conflict of Interest Statement}

The authors have no conflicts of interest to declare.

\section{Funding Sources}

The authors disclosed receipt of the following financial support for the research: Indian Council of Medical Research (ICMR) under the Junior Research Fellowship (JRF) Scheme, 2013 [20072].

\section{Author Contributions}

Shiny Lizia M. conducted the study, analysed the data and wrote the manuscript. Dr.Hemamalini A.J. and Dr. Latha Ravichandran corrected and revised the article critically for important intellectual content and approved the version to be published.

\section{Data Availability Statement}


All data generated and analyzed during this study are included in this article and its supplementary material files. Further enquiries can be directed to the corresponding author [AJH].

\section{References}

1. Rushing A, Sommer EC, Zhao S, Po'e EK, Barkin SL. Salivary epigenetic biomarkers as predictors of emerging childhood obesity. BMC Med Genet. 2020;21(1):34.

2. Lizia S Hemamalini AJ. Appetite Regulatory Hormones - A Novel Target for Prevention, Management, and Treatment of Obesity. Clin Med Rev in Therap. 2016;8 7-13

3. Zolotukhin S. Metabolic hormones in saliva: origins and functions. Oral Dis. 2013;19(3):219-29.

4. Yoshizawa JM, Schafer CA, Schafer JJ, Farrell JJ, Paster BJ, Wong DT. Salivary biomarkers: toward future clinical and diagnostic utilities. Clin micro rev. 2013;26(4), 781-791.

5. Gil-Campos M, Aguilera CM, Ramirez-Tortosa MC, Cañete R, Gil A. Fasting and postprandial relationships among plasma leptin, ghrelin, and insulin in prepubertal obese children. Clin Nutr. 2010;29(1):54-9.

6. Pandis N. Case-Control Studies: Part-I. Statistics And Research Design. 2014;146,2, P266-267,

7. Lean ME, Malkova D. Altered gut and adipose tissue hormones in overweight and obese individuals: cause or consequence? Int J Obes (Lond). 2016;40(4):622-632.

8. Belfort-DeAguiar R, Seo D. Food Cues and Obesity: Overpowering Hormones and Energy Balance Regulation. Curr Obes Rep. 2018;7(2):122-129.

9. Thanakun S, Watanabe H, Thaweboon S, Izumi Y. Comparison of salivary and plasma adiponectin and leptin in patients with metabolic syndrome. Diabetol Metab Syndr. 2014;6(1):19.

10. Li B-B, et al. Expression of ghrelin in human salivary glands and its levels in saliva and serum in Chinese obese children and adolescents. Arch. of Oral Bio. 2010;1-6.

11. Rambhojan C, Bouaziz-Amar E, Larifla L, et al., Ghrelin, adipokines, metabolic factors in relation with weight status in school-children and results of a 1-year lifestyle intervention program. Nutr Metab (Lond). 2015;12:43.

12. Mantovani RM, Rocha NP, Magalhães DM, Barbosa IG, Teixeira AL, Simões e Silva AC. Early changes in adipokines from overweight to obesity in children and adolescents. J Pediatr (Rio J). 2016;92:62430.

13. Ko BJ, Lee M, Park HS, Han K, Cho GJ, Hwang TG, et al., Elevated vaspin and leptin levels are associated with obesity in prepubertal Korean children. Endocr J. 2013;60:609--16.

14. Goodson JM, Kantarci A, Hartman M-L, Denis GV, Stephens D, et al., Metabolic Disease Risk in Children by Salivary Biomarker Analysis. PLoSONE 2014;9(6): e98799.

15. Tschöp M, Weyer C, Tataranni PA, Devanarayan V, Ravussin E, Heiman ML. Circulating ghrelin levels are decreased in human obesity. Diabetes. 2001;50(4):707-9.

16. Asayama $\mathrm{K}$ et al., Decrease in Serum Adiponectin Level Due to Obesity and Visceral Fat Accumulation in Children. Obesity Research. 2003;11: 1072-1079. 
17. Winer JC, Zern TL, Taksali SE, Dziura J, Cali AM, Wollschlager M, Seyal AA, Weiss R, Burgert TS, Caprio S. Adiponectin in childhood and adolescent obesity and its association with inflammatory markers and components of the metabolic syndrome. J Clin Endocrinol Metab. 2006;91(11):441523.

18. Weiss R et al., Low Adiponectin Levels in Adolescent Obesity: A Marker of Increased Intramyocellular Lipid Accumulation. J Clin Endocrinol Metab. 2003;88(5):2014-2018.

19. Soliman AT, Yasin M, Kassem A. Leptin in pediatrics: A hormone from adipocyte that wheels several functions in children. Indian J Endocrinol Metab. 2012;16(Suppl 3):S577-S587.

20. Hagen EW, Starke SJ, Peppard PE. The Association between Sleep Duration and Leptin, Ghrelin, and Adiponectin Among Children and Adolescents. Curr Sleep Medicine Rep. 2015; 1:185-194 (2015).

\section{Tables}

Table 1

Mean Baseline Characteristics of the Subjects

\begin{tabular}{|c|c|c|c|}
\hline \multirow[t]{4}{*}{ Characteristics } & \multicolumn{2}{|c|}{ Groups ( $N=166)$} & \multirow[t]{4}{*}{ p-value } \\
\hline & Obese (Case) & Non-obese (Control) & \\
\hline & (Mean \pm S.D.) & (Mean \pm S.D.) & \\
\hline & $(n=83)$ & $(n=83)$ & \\
\hline Age & $8.67 \pm 1.09$ & $8.61 \pm 1.03$ & 0.716 \\
\hline Height & $133.76 \pm 6.23$ & $135.07 \pm 7.14$ & 0.211 \\
\hline Weight & $38.74 \pm 5.59$ & $29.62 \pm 5.73$ & $0.000^{\star *}$ \\
\hline WAZ & $1.72 \pm 0.40$ & $0.07 \pm 0.94$ & $0.000^{\star *}$ \\
\hline HAZ & $0.12 \pm 0.46$ & $0.40 \pm 0.75$ & $0.024^{\star}$ \\
\hline BAZ & $2.11 \pm 0.28$ & $-0.13 \pm 0.93$ & $0.000 * *$ \\
\hline${ }^{\star} * P<0.01 ;{ }^{*} P<0$. & & & \\
\hline
\end{tabular}


Table 2

Salivary Metabolic Hormones as Biomarkers of Childhood Obesity $(\mathrm{N}=166)$

\begin{tabular}{|lll|}
\hline Variables & Odds Ratio & p-value \\
\hline Age & $1.06(0.79-1.41)$ & 0.714 \\
\hline Gender - Female & $1(0.55-1.84)$ & 1.000 \\
\hline Height & $0.97(0.93-1.02)$ & 0.211 \\
\hline Weight & $1.33(1.22-1.45)$ & $0.000^{\star *}$ \\
\hline WAZ & $132.99(20.86-847.86)$ & $0.000^{* *}$ \\
\hline HAZ & $0.47(0.28-0.80)$ & $0.005^{*}$ \\
\hline Ghrelin & $0.001(0-0.01)$ & $0.000^{* *}$ \\
\hline Leptin & $1.03(1.02-1.04)$ & $0.000^{* *}$ \\
\hline Adiponectin & $0.99(0.99995-0.99998)$ & $0.000^{* *}$ \\
\hline Insulin & $1.085(1.054-1.117)$ & $0.000^{* *}$ \\
\hline$* * P<0.01 ;{ }^{*} \mathrm{P}<0.05$ & & \\
\hline
\end{tabular}

Figures 
Figure 1. STROBE Study Chart

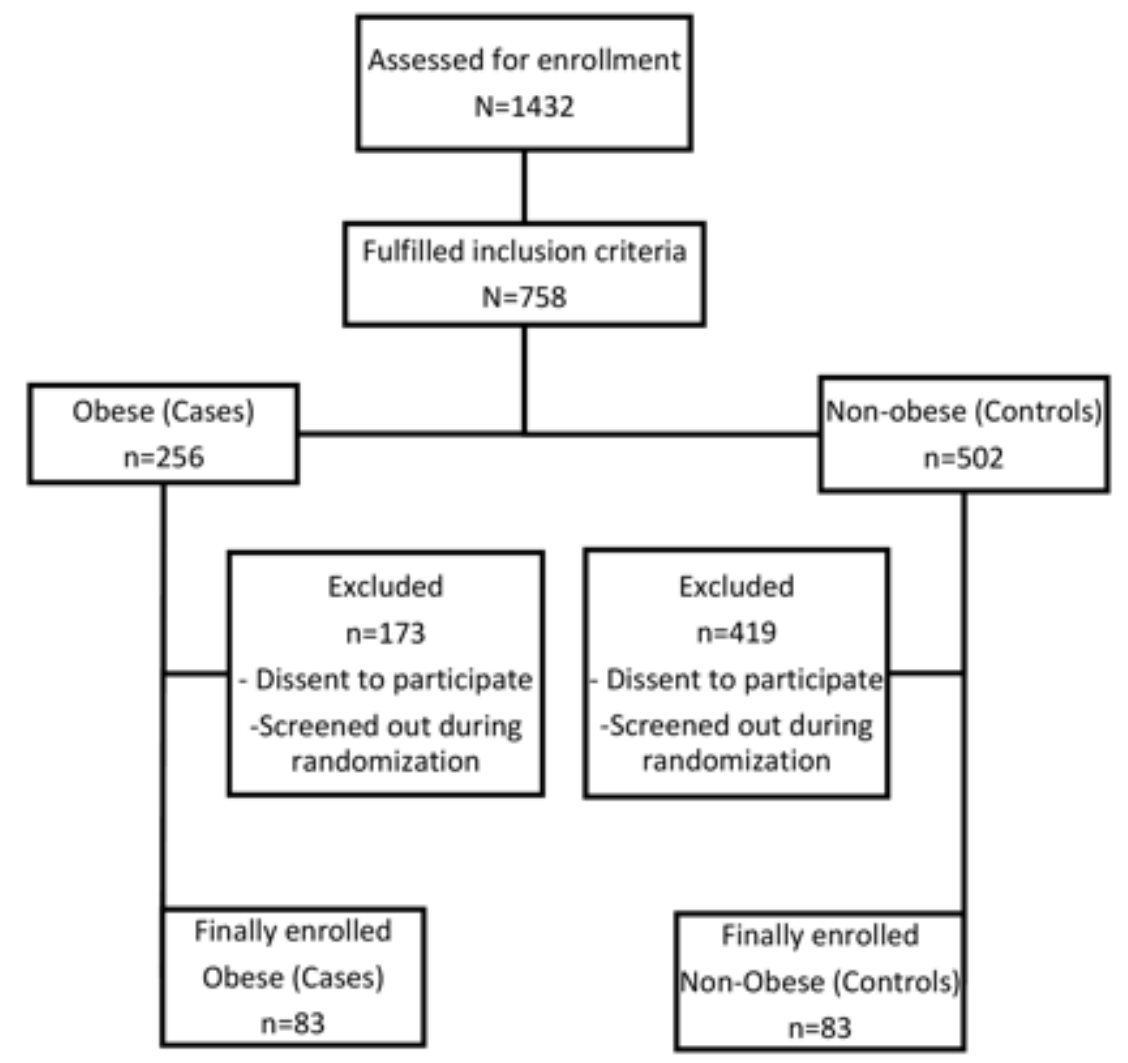

Figure 1

STROBE Study Chart 


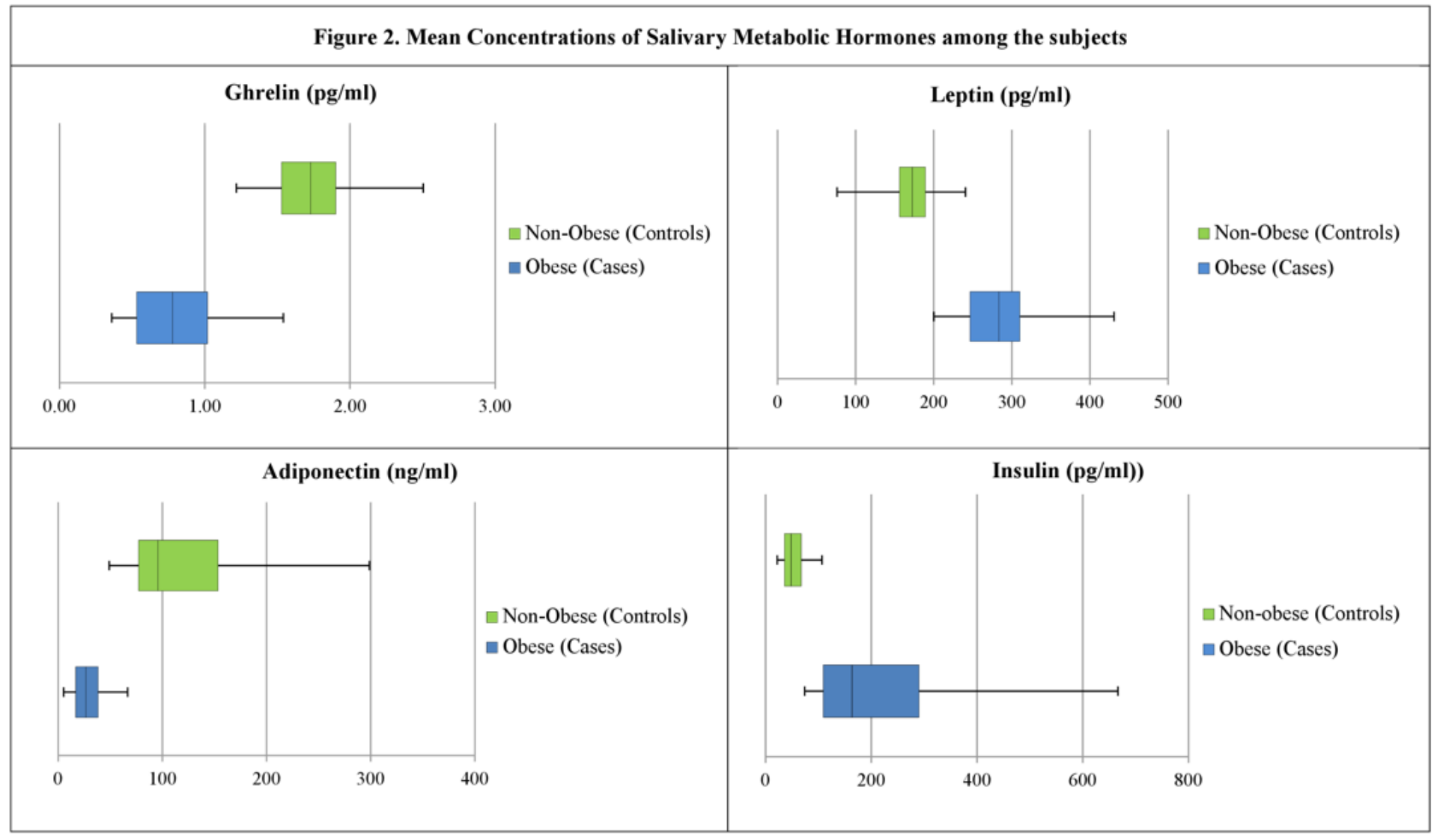

Figure 2

Mean Concentrations of Salivary Metabolic Hormones among the subjects. 